\title{
Molecular Identification, Isolation and Evaluation of Persian Gulf Actinomycetes as Candidates of Cytotoxic Metabolites Against Breast Cancer
}

\author{
Aref Bahri ${ }^{1,2}$, Elham Moazamian ${ }^{2,}{ }^{,}$, Negar Azarpira ${ }^{3}$ \\ ${ }^{1}$ Department of Microbiology, School of Basic Sciences, Science and Research Branch, \\ Islamic Azad University, Shiraz, Iran \\ ${ }^{2}$ Department of Microbiology, College of Science, Agriculture and Modern \\ Technologies, Shiraz Branch, Islamic Azad University, Shiraz, Iran \\ ${ }^{3}$ Organ Transplant Research Center, Shiraz University of Medical Sciences, Shiraz, \\ Iran \\ ${ }^{*}$ Corresponding author: Elham Moazamian, Department of Microbiology, College of \\ Science, Agriculture and Modern Technologies, Shiraz Branch, Islamic Azad University, \\ Shiraz,Iran.E-mail:moazamian@iaushiraz.ac.ir,elhammoazamian@gmail.com
}

DOI: $10.21859 /$ mci-01035

Submitted: 6 May 2017

Revised: 12 June 2017

Accepted: 23 June 2017

ePublished: 1 July 2017

Keywords:

Persian Gulf

Marine Actinomycetes

Anti-cancer

Secondary Metabolites

\begin{abstract}
Introduction: Marine actinomycetes have a great potential to produce unique bioactive compounds due to their special adaptation in the harsh ocean environment. The current study aimed to isolate anti-cancer compounds producing actinomycetes from sediments of Harra forests of the Persian Gulf and investigate their potential as anti-breast cancer metabolites.

Methods: In the current study, 40 sediment samples of Harra forests of the Persian Gulf were collected. Samples were diluted and cultured in a starch casein agar selective medium. The strains were isolated and purified, using morphological and microscopic methods. Forty strains were cultured in a starch casein broth and the metabolites were extracted using ethyl acetate. The produced metabolites were extracted from active strains and their cytocidal activities were evaluated against the breast cancer cell line. Finally, effective-metabolites-producing bacteria were identified using the molecular method.

Results: Of the sediments, 186 strains were isolated and identified. Results showed that the isolates had cytocidal activities against the breast cancer cell line. The results also revealed that the $2 \mathrm{HP}$ and $4 \mathrm{HP}$ strains showed more anti-cancer activities than the others. Results of this study showed that sediments of Harra forests of the Persian Gulf were rich in active actinomycetes, which can be used in the production of new anticancer compounds.

Conclusions: The obtained results give evidence that it is essential to scrutinize these marine microorganisms, which have a great potential to be used in the pharmaceutical fields, in search for new drugs.
\end{abstract}

(C) 2017. Multidisciplinary Cancer Investigation

\section{INTRODUCTION}

Actinomycetes are filamentous, Gram-positive, free-living, and saprophyte bacteria that sometimes are symbiotic with plants. These bacteria can be isolated from all ecosystems including soil, water, marine sediments, and even hot waters [1]. Actinomycetes form about $10 \%$ of the population of bacteria in the seabed sediment. Microorganisms of marine habitats are considered as amazing sources of strong bioactive materials [2]. Actinomycetes have a great potential to produce secondary metabolites such as antibiotics, enzymes, herbicides, anti-cancer materials, and other useful compounds [3, 4]. About 23000 kinds of active secondary metabolites are produced by microorganisms, and actinomycetes produce more than $75 \%$ of different antibiotics and antimicrobial agents known as new active sources [5]. In recent years, due to the need for new drugs, marine microorganisms, with their potential to produce unique metabolites, are considered as new sources [6]. Cancer is one of the most serious issues that threaten human life and breast cancer is the second leading cause of death in women. Many marine natural products with anti-cancer effects are derived from marine Actinobacteria. These metabolites play an important role in identifying drug compounds. At present, few studies are conducted on 
the anti-cancer role of bioactive compounds derived from marine Actinobacteria [7]. In the past half century, thousands of antibiotics are successfully used to treat microbial diseases. Therefore, it is no surprise that a large number of bacteria and tumor cells show resistance against antimicrobial agents. In the past, the solution to this problem was to use new anti-cancer drugs and antimicrobial sources. However, in recent years, the use of these antibiotics is drastically reduced due to the exhaustion of conventional resources. Hence, scarce resources such as marine Actinobacteria raised attention. These bacteria are capable of producing anti-cancer bioactive metabolites which are isolated from the marine environment [8]. In the current study, actinomycetes were isolated from the mangrove ecosystem of the Naiband Bay in Assaluyeh located in the Persian Gulf, and their metabolites' cytocidal effects on breast cancer were evaluated.

\section{METHODS}

\section{Sampling and Isolation of Actinomycetes}

To isolate actinomycetes in the current study, geographic coordinates of the Naiband Bay located in Assaluyeh, Bushehr province, were obtained using the GPS in June 2015. Forty points were determined with 500 meters distance from each other. Coordinates of each sample are shown in Table 1.

Collected samples were put in sampling containers and transported to the laboratory in the shortest time. Then, samples were incubated for 1 hour at $55^{\circ} \mathrm{C}$ to reduce vegetative forms of actinomycetes and other bacteria [9]. The serial dilution method was used to isolate actinomycetes and various dilutions obtained from sediment samples were cultured for 7 days in a starch-casein agar medium (including $15 \mathrm{~g}$ of agar, $1 \mathrm{~g}$ of potassium phosphate, $2 \mathrm{~g}$ of sodium chloride, $2 \mathrm{~g}$ of potassium nitrate, $0.3 \mathrm{~g}$ of casein, $0.05 \mathrm{~g}$ of magnesium sulfate 7-water, $0.01 \mathrm{~g}$ of ferrous sulfate 7 -water, and $0.02 \mathrm{~g}$ of calcium carbonate in a total volume of 1 liter of filtered sea water to increase the isolation rate of actinomycetes) in $7.5 \mathrm{pH}$, and were incubated at $28^{\circ} \mathrm{C}$. To prevent the growth of fungi and bacteria, $25 \mu \mathrm{g} / \mathrm{L}$ nystatin and $10 \mu \mathrm{g} / \mathrm{L}$ nalidixic acid were respectively added to the culture medium. After 7 days, colonies of actinomycetes were selected and isolated based on the specific characteristics [10].

\section{Extraction of Metabolites from Actinomycetes Iso- lates}

To extract the anti-cancer metabolites, active isolates of actinomycetes were inoculated in starch-casein broth liquid medium. To produce anti-cancer compounds, Erlenmeyer flasks were put for 7 days at $28^{\circ} \mathrm{C}$ in a shaking incubator at $180 \mathrm{rpm}$ [3]. To isolate the mycelia from the liquid phase, the culture medium containing metabolites was passed through syringe filters ( $45 \mu \mathrm{m}$ size) and centrifuged at $5000 \mathrm{rpm}$ for 10 minutes. Due to the solubility of antibiotic compounds in organic solvents, an ethyl acetate organic solvent was used to extract the anti-cancer metabolites. Ethyl acetate was added by an equal volume of supernatant obtained from each isolate. The organic phase, containing antibiotic compounds, was isolated by a decanter and condensed by heat. The extracted metabolites were used to investigate the anti-cancer activity of the isolates on the breast cancer cell line $[11,12]$.

Table 1: Coordinates of Sampling Stations

\begin{tabular}{|c|c|c|}
\hline Station & East longitude & North latitude \\
\hline 1 & 3039567 & 662318 \\
\hline 2 & 3039446 & 662457 \\
\hline 3 & 3039326 & 662651 \\
\hline 4 & 3039204 & 662762 \\
\hline 5 & 3039082 & 663232 \\
\hline 6 & 3038964 & 663232 \\
\hline 7 & 3038850 & 664498 \\
\hline 8 & 3038735 & 664498 \\
\hline 9 & 3038617 & 664911 \\
\hline 10 & 3038499 & 665270 \\
\hline 11 & 3038378 & 665436 \\
\hline 12 & 3038256 & 665548 \\
\hline 13 & 3038135 & 665659 \\
\hline 14 & 3039444 & 662265 \\
\hline 15 & 3039322 & 662403 \\
\hline 16 & 3039201 & 662570 \\
\hline 17 & 3040926 & 662657 \\
\hline 18 & 3040805 & 662768 \\
\hline 19 & 3038837 & 662959 \\
\hline 20 & 3038817 & 663180 \\
\hline 21 & 3038598 & 663484 \\
\hline 22 & 3038598 & 664035 \\
\hline 23 & 3038366 & 664530 \\
\hline 24 & 3038265 & 666179 \\
\hline 25 & 3038129 & 665248 \\
\hline 26 & 3038008 & 665386 \\
\hline 27 & 3037887 & 665553 \\
\hline 28 & 3037766 & 665692 \\
\hline 29 & 3039196 & 662185 \\
\hline 30 & 3040923 & 662382 \\
\hline 31 & 3038956 & 662628 \\
\hline 32 & 3038835 & 662822 \\
\hline 33 & 3038715 & 663016 \\
\hline 34 & 3038594 & 663154 \\
\hline 35 & 3038472 & 663293 \\
\hline 36 & 3038353 & 663597 \\
\hline 37 & 3038233 & 663846 \\
\hline 38 & 3038112 & 664012 \\
\hline 39 & 3037992 & 664178 \\
\hline 40 & 3037837 & 664510 \\
\hline
\end{tabular}




\section{Cell Culture}

Cell line 4T1 was purchased from the Pasteur Institute of Iran and cultured in the medium RPMI 1640 (Biosera, England) enriched with $10 \%$ fetal bovine serum and streptomycin (100 $\mu \mathrm{g} / \mathrm{mL})$ and penicillin $(100 \mathrm{U} / \mathrm{mL})$. Cells were incubated at $37^{\circ} \mathrm{C}, 95 \%$ humidity, and $5 \%$ carbon dioxide [13].

\section{Investigation of Cytocidal Features}

To evaluate the anti-cancer properties of metabolites, cells were cultured for 24 hours in 96-well plates. For this purpose, a $90-\mathrm{mL}$ culture medium containing $2 \times 10^{4}$ cells and 10 microliters of isolated metabolites (concentration of 1 $\mathrm{mg} / \mathrm{mL}$ ) were added to each well. The test for the positive control samples was conducted adding Triton X-100, negative control (no metabolites), and ethyl acetate control in 24 hours of incubation. To assess cytotoxicity, the MTT assay was used. This method is based on reviving solution dimethyl diphenyl tetrazolium bromide color to an insoluble product of purple formazan by the mitochondrial reductase enzyme activity in the living cells, and the number of living cells can be detected from the nonliving ones. The MTT solution at a concentration of $5 \mathrm{mg} / \mathrm{mL}$ was prepared in a RPMI medium. The MTT assay was used to investigate the cytocidal activity of metabolites on cell lines, and the results were read by the enzyme-linked immunosorbent assay (ELISA) reader device at 430 and $692 \mathrm{~nm}$ wavelengths [14]. Isolated metabolites, with the highest cytotoxicity, were selected and their cytotoxicity effects were evaluated in different concentrations $\left(10^{-1}, 10^{-2}, 10^{-3}, 10^{-4}, 10^{-5}\right.$ and 10 $\left.{ }^{6}\right)$ within 24 and 48 hours $[14,15]$.

\section{Observing Cytopathic Effects}

To view the cytopathic effects of metabolites on the breast cancer cell line, imaging was performed using an inverted microscope.

\section{Molecular Identification of Actinomycetes Isolates Using 16S rDNA}

DNA extraction was conducted using a bacterial DNA extraction kit according to the manufacturer's instructions (Yekta Teb Tajhiz, Iran). Polymerase chain reaction (PCR) was performed in a total volume of $25 \mu \mathrm{L}$ containing $1 \mathrm{mM}$ $\mathrm{MgCl}_{2}, 2 \mathrm{U}$ Taq polymerase (Cinnagen, Iran), $75 \mu \mathrm{M}$ of each dNTP, $1 \mu \mathrm{L}$ of each primer (with concentration of $10 \mathrm{pmol}$ ) containing forward primer (5'AGAGTTTGATCCTGGCTCAG 3') $27 \mathrm{f}$ and reverse primer (5' GGTTACCTTGTTACGACTT 3') 1492r, $12.5 \mu \mathrm{L} 10 \mathrm{X}$ PCR (Cinnagen, Iran), and 1 $\mu \mathrm{L}$ of template DNA. In this technique, to begin the amplification process, a thermal cycler PCR machine (BioRAD) was set at $94^{\circ} \mathrm{C}$ for 1 minute. Then, 35 cycles of PCR were amplified at $94^{\circ} \mathrm{C}$ for 1 minute, $52^{\circ} \mathrm{C}$ for 30 seconds, and $72^{\circ} \mathrm{C}$ for 1 minute. Finally, elongation was done for 4 minutes at $72^{\circ} \mathrm{C}$. Then, to ensure the amplification of the $16 \mathrm{~S}$ rDNA gene, electrophoresis was conducted on $1 \%$ agarose gel, containing
Tris/Borate/EDTA (TBE) $1 \mathrm{X}$ buffer for 60 minutes at $90 \mathrm{~V}$. Finally, a UV transilluminator was used to observe the results. At last, the PCR final product was sent to the South Korean Macrogen Co. for sequencing [16].

\section{Statistical Analysis}

One-way ANOVA with post hoc test was employed to compare numeric data among different concentrations.

\section{RESULTS}

Isolation of Actinomycetes from Marine Sediments

Among 40 sediment samples, 186 marine actinomycetes were isolated based on the colony, morphological, and microscopic characteristics. The morphological characteristics of actinomycetes are rough and dry looking, and due to their closeness to fungi, their appearance is similar to them (Figure 1).

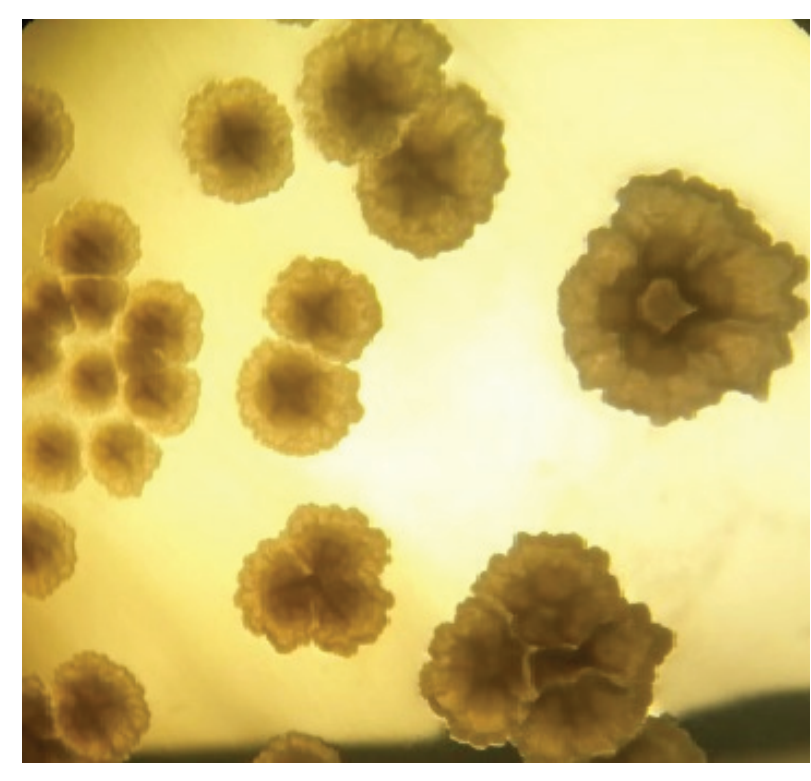

Figure 1: Colonies of the Isolated Actinomycetes

\section{Identification of Actinomycetes with Highest Per- centage of Cytotoxicity}

At this stage, metabolites of 186 isolates at a concentration of $1 \mathrm{mg} / \mathrm{mL}$ were treated on the breast cancer cell lines. The highest cytotoxic effects were observed in samples of $2 \mathrm{HP}$ (34.27\%) and 4HP (33.50\%). Results of the metabolites extracted from samples at different concentrations showed that increased concentration of $2 \mathrm{HP}$ and $4 \mathrm{HP}$ led to decrease survival of cancer cells; hence, in the $2 \mathrm{HP}$ sample, the survival rate of cancer cells was decreased from $99.2 \%$ in 0.0001 $\mathrm{mg} / \mathrm{mL}$ concentration to $65.66 \%$ in $100 \mathrm{mg} / \mathrm{mL}$, within 48 hours. In addition, in the $4 \mathrm{HP}$ sample, the cell survival rate was decreased from $99.98 \%$ in $0.0001 \mathrm{mg} / \mathrm{mL}$ concentration to $63.8 \%$ in $100 \mathrm{mg} / \mathrm{mL}$ (Figure 2). 


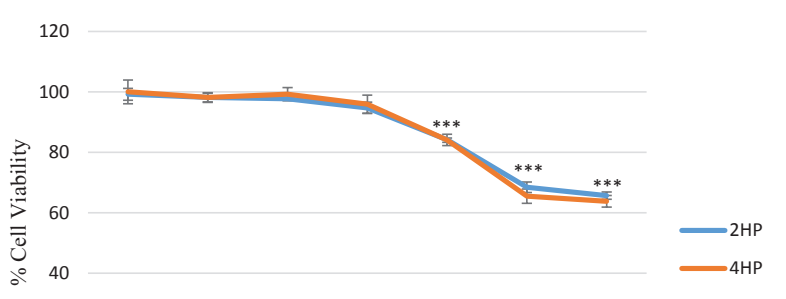

20

0

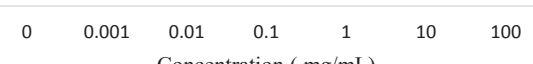

Concentration $(\mathrm{mg} / \mathrm{mL})$

Figure 2: Comparison of the Diagram of Changes of the Samples (2HP and 4HP) at Different Concentrations at 48 Hours.

The diagram showed that survival of cancer cells significantly decreased at the concentrations of 1,10 , and $100 \mathrm{mg} / \mathrm{mL} .{ }^{* * *}$ : $\mathrm{P}$ < 0.001

\section{Imaging by an Inverted Microscope}

Cytopathic effects of 2HP and 4HP actinomycetes metabolites were observed using an inverted microscope, and imaged after 48 hours. The results showed that metabolites caused morphological changes associated with apoptosis in 4T1 cells; therefore, in the treated cells, the structure of the spindle-shaped cells changed and it was observed as a spherical or collapsed form (Figure 3).
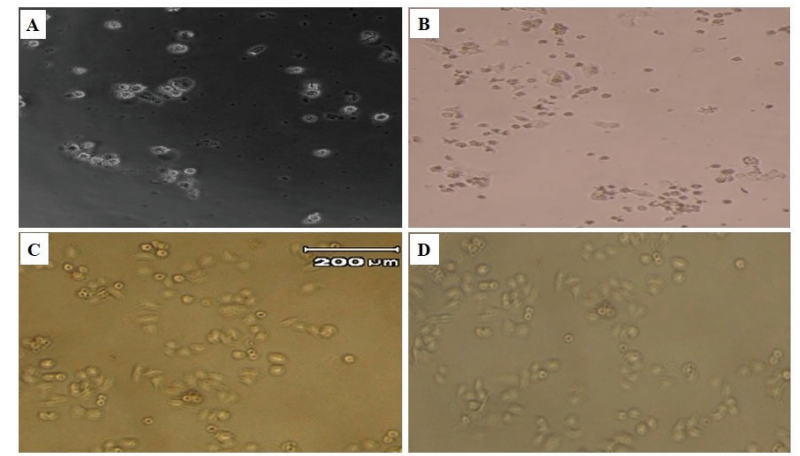

Figure 3: Cytopathic Effects of 2HP and 4HP Isolated Metabolites, Compared with the Negative and Ethyl Acetate Controls on the Breast Cancer Cell Line after 48 Hours. A) Negative Control, B) 2HP Isolate, C) 4HP Isolate, D) Ethyl Acetate Control

\section{$16 S$ rDNA Sequencing of the Selected Strains}

The 16S rDNA gene of the selected strains was sequenced. The partial sequence of the $16 \mathrm{~S}$ rDNA gene was amplified using the BLAST software and compared with the other bacteria. The results showed that the sequence of the selected $2 \mathrm{HP}$ and $4 \mathrm{HP}$ isolates obtained based on the sequence homology of $98 \%$ had a genetic similarity with the streptomyces strains.

\section{DISCUSSION}

It is important to find new antitumor agents to treat diseases and cancers. Streptomyces are one of the most important microorganism producing antitumor agents. Wide biodiversity in the seas and oceans is a sign of the diversity of chemicals in marine environments. Since marine organisms, especially actinomycetes, have the largest metabolic and genomic diversity, there are efforts to discover marine actinomycetes as new drug sources. With limited screenings dedicated to marine actinomycetes until today, the discovery of new secondary metabolites produced by the marine actinomycetes of the soil type became higher. According to the increasing trend of resistance against antibiotics in the bacteria and chemotherapy drug problems, the use of marine natural compounds attracted the scientists' attention [1]. Therefore, oceans are a huge library of natural unique compounds and products, and promising and surprising bioactive materials that can be never found on the earth. Recently, it is demonstrated that the ocean floor is an ecosystem with unique forms of actinomycetes in itself. It seems that actinomycetes are distributed in all over the oceans; therefore, they are found in tidal regions, ocean sediments, seawater, mangroves, fishes, jellyfishes, seaweeds, and sponges. Since the marine environmental conditions are totally different from that of drought, it seems that marine actinomycetes should also be very different from their soil forms. Hence, marine actinomycetes are able to produce new antibiotics and bioactive compounds $[16,17]$. In the present study, Persian Gulf was selected as a marine ecosystem to evaluate the anti-cancer activity of actinomycetes in the sediment samples which can be cultured using above mentioned methods. Mangrove areas located in the Naiband Bay in Assaluyeh were selected as sampling stations. Sampling was mostly done close to the roots of mangrove trees to increase bacterial isolation, and significant colonies of actinomycetes were isolated from them, and the primary identification was performed based on the morphological features of the colony. In the current study, 186 actinomycete species were isolated from the Naiband. Ravi Kumar et al., (2012) collected actinomycete isolates from the mangrove ecosystem, and reported that the mangrove ecosystem was a good place to isolate actinomycetes [14]. In the present study, the effects of purified cytocidal metabolites were examined at a concentration of $1 \mathrm{mg} / \mathrm{mL}$ for 48 hours and the results indicated the inhibition of cancer cells proliferation. Kumar et al., (2013) isolated different actinomycete strains from the Indian Ocean, and reported that the isolated strains had inhibitory effects on the growth of breast cancer cell lines [18]. Moreover, Valliappan et al., (2013) reported that the actinomycetes extract showed a cytocidal effect on cancer cells and breast cancer cell lines [19], consistent with the results of the present study indicating the cytotoxic effect of actinomycetes extract on the breast cancer cell line. Jayamadhuri and Krishna (2013) isolated actinomycete strains from a tidal zone, and reported that actinomycete extracts had cytotoxic effects on the growth of breast cancer cell lines [20]. Fedorov conducted a study on marine actinomycetes in 2013 and 
reported that actinomycetes had a great potential in the treatment of cancer consistent with the result of the present study [21]. Ravikumar et al., (2012) reported that the anti-cancer property of cell-free extracts from actinomycete isolates might be due to the presence of the active secondary metabolites such as alkaloids and quinines [14]. Manivasagan et al., (2014) reported that the marine environmental conditions are extremely different from that of the terrestrial ones. The marine microbes have different characteristics, and therefore, might produce different types of bioactive compounds in their challenging living conditions [22]. According to above mentioned effects of actinomycete extracts, further studies seem to be necessary to identify possible mechanisms of apoptosis induction by the metabolites extracted from the actinomycetes on the cancer cell lines. Studies on the effects of actinomycete metabolites on human normal cells need to be fully understood. Moreover, the susceptibility of actinomycete metabolites should be investigated in vivo.

In general, according to drug resistance and problems caused by the side effects of chemotherapy in patients with cancer, identification of new effective drugs including metabolites from actinomycetes may play an important role in further studies on cancer treatment. It is hoped that with a greater focus on marine natural resources, cancer and other diseases can be controlled.

\section{ACKNOWLEDGMENTS}

The authors would like to thank the Organ Transplant Research Center of Shiraz University of Medical Sciences for cell culture.

This research had no funding and it is based on an MSc thesis.

\section{CONFLICT OF INTEREST}

The authors declared no conflict of interest.

\section{ETHICS APPROVAL}

Not applicable.

\section{REFERENCES}

1. Abdi Soofiani S, Dehnad AR, Nahaei MR, Parsa Yegane L, Barzegari A, Maleki Kakolr H. Molecular Identification of Streptomyces Spp. with Antibacterial Activity Isolated from East Azerbaijan Soils. J Tabriz Univ Med Sci. 2011;33(2):4956.

2. Safaeian S, Nouhi A, Oryan S. Studies on cytotoxic activity of marine Actionomycetes from Persian Gulf on artemia franciscana and artemia Urmiana. J Agricult Sci Nat Resourc. 2005;11(4):133-9.

3. Valli S, Suvathi SS, Aysha OS, Nirmala P, Vinoth KP, Reena A. Antimicrobial potential of Actinomycetes species isolated from marine environment. Asian Pac J Trop Biomed. 2012;2(6):469-73. DOI: 10.1016/S2221-1691(12)60078-1 PMID: 23569952

4. Petrova DH, Shishkov SA, Vlahov SS. Novel thermostable serine collagenase from Thermoactinomyces sp. 21E: purification and some properties. J Basic Microbiol. 2006;46(4):275 85. DOI: 10.1002/jobm.200510063 PMID: 16847831

5. Dehnad A, Bakhshi R, Yeganeh Parsa L, Sefidan Monadi A, Montazam S, Abdi Soufiani S. Screening of Streptomycetes with Antibacterial Activity from Soil Samples of Azarbaijan Regions of Iran. J Microb Biotechnol. 2009;1(1):18-22.

6. Sharma H, Parihar L. Antifungal activity of extracts obtained from Actinomycetes. J Yeast Fung Res. 2010;1(10):197-200.

7. Ravikumar S, Gnanadesigan M, Thajuddin N, Chakkaravarthi VD, Banerjee B. Anticancer property of sponge associated actinomycetes along Palk Strait. J Pharm Res. 2010;3(10):24157.

8. Demin S. Marine streptomyces as a novel source of bioactive substances. World J Microbiol Biotechnol.2009;26(12):2123 39.

9. Takizawa M, Colwell RR, Hill RT. Isolation and diversity of actinomycetes in the chesapeake bay. Appl Environ Microbiol. 1993;59(4):997-1002. PMID: 16348922

10. Das S, Ward LR, Burke C. Screening of marine Streptomyces spp. for potential use as probiotics in aquaculture. Aquacult. 2010;305(1-4):32-41. DOI: $10.1016 /$ j.aquaculture.2010.04.001

11. Jianyou L, Jianrong X, Yongheng C. Isolation and identification of two marine-derived Streptomyces from marine mud of coast and offshore Zhuhai, and bioactive potential for plant pathogenic fungi. Afr J Biotechnol. 2011;10(56):11855-60.

12. Singh LS, Sharma H, Talukdar NC. Production of potent antimicrobial agent by actinomycete, Streptomyces sannanensis strain SU118 isolated from phoomdi in Loktak Lake of Manipur, India. BMC Microbiol. 2014;14(2):278. DOI: 10.1186 s12866-014-0278-3 PMID: 25406714

13. Moosav M, Ghanbarvand F, Dehnad A. Growth Inhibition and Induction of Apoptosis by Ether Soluble Metabolitesof Streptomyces sp. ABRIINW 111 in Human Myeloid Leukemia K562 Cell. J Cell Tissue. 2011;2(3):225-34.

14. Ravikumar S, Suganthi P, Moses F. Crude bioactive compounds of actinomycetes from manakkudy mangrove sediment. J Pharm Res. 2011;4(3):877-9.

15. S B, Moazamian E, Azarpira N. Cytocidal effects of Bacillus thuringiensis crystal protein on mice breast cancer cell line in in vitro condition. J Cell Tissue. 2017;3:243-50.

16. Bandari Z, Mozamian E, Azarpira N. Investigating the Cytotoxic Effects of Persian Gulf Marine Actinomycetes Protease on Blood Cancer Cell Line. Basic Clin Cancer Res. 2017;8(4):3-14.

17. Bernan VS, Greenstein M, Maiese WM. Marine microorganisms as a source of new natural products. Adv Appl Microbiol. 1997;43:57-90. PMID: 9097412

18. Kumar RS, Rajkapoor B, Perumal P. In vitro and in vivo anticancer activity of Indigofera cassioides Rottl. Ex. DC. Asian Pac J Trop Med. 2011;4(5):379-85. DOI: 10.1016/S1995 7645(11)60108-9 PMID: 21771681

19. Jeong SY, Shin HJ, Kim TS, Lee HS, Park SK, Kim HM. Streptokordin, a new cytotoxic compound of the methylpyridine class from a marine-derived Streptomyces sp. KORDI-3238. J Antibiot (Tokyo). 2006;59(4):234-40. DOI: 10.1038/ ja.2006.33 PMID: 16830891

20. Jayamadhuri R, Krishna K. In vitro Anti-cancer Activity of Marine Bacteria Isolated from Andhra Pradesh and Tamil Nadu Coastal Regions. Int J Chem Environ Biol Sci. 2013;2:2320-4079.

21. Fedorov SN, Ermakova SP, Zvyagintseva TN, Stonik VA Anticancer and cancer preventive properties of marine polysaccharides: Some results and prospects. Marine Drugs. 2013;11(12):4876-901.

22. Manivasagan P, Venkatesan J, Sivakumar K, Kim SK. Pharmaceutically active secondary metabolites of marine actinobacteria. Microbiol Res. 2014;169(4):262-78. DOI: $10.1016 / \mathrm{j}$. micres.2013.07.014 PMID: 23958059 